\title{
THE 2dF GALAXY REDSHIFT SURVEY: CONSTRAINTS ON COSMIC STAR FORMATION HISTORY FROM THE COSMIC SPECTRUM
}

\author{
Ivan K. Baldry, ${ }^{1}$ Karl Glazebrook, ${ }^{1}$ Carlton M. Baugh, ${ }^{2}$ Joss Bland-Hawthorn, ${ }^{3}$ Terry Bridges, ${ }^{3}$ \\ Russell Cannon, ${ }^{3}$ Shaun Cole, ${ }^{2}$ Matthew Colless, ${ }^{4}$ Chris Collins, ${ }^{5}$ Warrick Couch, ${ }^{6}$ Gavin Dalton, ${ }^{7}$ \\ Roberto De Propris, ${ }^{6}$ Simon P. Driver, ${ }^{8}$ George Efstathiou, ${ }^{9}$ Richard S. Ellis, ${ }^{10}$ Carlos S. Frenk, ${ }^{2}$ \\ Edward Hawkins, ${ }^{11}$ Carole Jackson, ${ }^{4}$ Ofer Lahav, ${ }^{9}$ Ian Lewis, ${ }^{7}$ Stuart Lumsden, ${ }^{12}$ Steve Maddox, 11 \\ Darren S. Madgwick, ${ }^{9}$ Peder Norberg, ${ }^{2}$ John A. Peacock, ${ }^{13}$ Bruce A. Peterson, ${ }^{4}$ \\ Will Sutherland, ${ }^{7}$ AND KeITH TAYLOR ${ }^{3}$ \\ Received 2001 October 31; accepted 2002 January 3
}

\begin{abstract}
We present the first results on the history of star formation in the universe based on the "cosmic spectrum," in particular the volume-averaged, luminosity-weighted, stellar absorption-line spectrum of presentday galaxies from the $2 \mathrm{dF}$ Galaxy Redshift Survey. This method is novel in that, unlike previous studies, it is not an estimator based on total luminosity density. The cosmic spectrum is fitted with models of population synthesis, tracing the history of star formation before the epoch of the observed galaxies, using a method we have developed that decouples continuum and spectral line variations and is robust against spectrophotometric uncertainties. The cosmic spectrum can only be fitted with models incorporating chemical evolution, and it indicates that there was a peak in the star formation rate (SFR) in the past of at least 3 times the current value and that the increase back to $z=1$, assuming it scales as $(1+z)^{\beta}$, has a strong upper limit of $\beta<5$. We find, in the general case, that there is some model degeneracy between star formation at low and high redshift. However, if we incorporate previous work on star formation at $z<1$, we can put strong upper limits on the star formation rate at $z>1$ : e.g., if $\beta>2$, then the SFR for $1<z<5$ scales as $(1+z)^{\alpha}$, with $\alpha<2$. This is equivalent to stating that no more than $80 \%$ of stars in the universe formed at $z>1$. Our results are consistent with the best-fit results from compilations of cosmic SFR estimates based on UV luminosity density, which yield $1.8<\beta<2.9$ and $-1.0<\alpha<0.7$, and are also consistent with estimates of $\Omega_{\text {stars }}$ based on the $K$-band luminosity density.
\end{abstract}

Subject headings: cosmology: miscellaneous — cosmology: observations — galaxies: evolution — stars: formation

On-line material: color figures

\section{INTRODUCTION}

The analysis of the comoving star formation rate (SFR) density as a function of redshift has been the subject of much recent work. The onset of large redshift surveys at $z<1$ (see, e.g., Lilly et al. 1996) and $z>3$ (see, e.g., Steidel

1 Department of Physics and Astronomy, Johns Hopkins University, Baltimore, MD 21218-2686.

${ }^{2}$ Department of Physics, University of Durham, South Road, Durham DH1 3LE, UK.

${ }^{3}$ Anglo-Australian Observatory, P.O. Box 296, Epping, NSW 2121, Australia.

${ }^{4}$ Research School of Astronomy and Astrophysics, Australian National University, Weston Creek ACT 2611, Australia.

${ }^{5}$ Astrophysics Research Institute, Liverpool John Moores University, Twelve Quays House, Birkenhead L14 1LD, UK.

${ }^{6}$ Department of Astrophysics, University of New South Wales, Sydney NSW 2052, Australia.

7 Department of Physics, University of Oxford, Keble Road, Oxford OX1 3RH, UK

${ }^{8}$ School of Physics and Astronomy, University of St. Andrews, North Haugh, St. Andrews, Fife KY6 9SS, UK.

${ }^{9}$ Institute of Astronomy, University of Cambridge, Madingley Road, Cambridge CB3 0HA, UK.

${ }^{10}$ Department of Astronomy, California Institute of Technology, Pasadena, CA 91125

${ }^{11}$ School of Physics and Astronomy, University of Nottingham, Nottingham NG7 2RD, UK.

${ }^{12}$ Department of Physics, University of Leeds, Woodhouse Lane, Leeds LS2 9JT, UK.

${ }^{13}$ Institute for Astronomy, University of Edinburgh, Royal Observatory, Blackford Hill, Edinburgh EH9 3HJ, UK. et al. 1999) has allowed the volumetric emission of luminosity in different bands to be traced with redshift. In particular, from these studies there is now good evidence of a rise in star formation rate by a factor of about 8 between $z=0$ and $z=1$ (Hogg 2001) and either a $z>1$ decline (Madau et al. 1996) or plateau (Pettini et al. 1998).

Most measurements of the SFR to date have been based on some type of luminosity density, which is thought on theoretical and/or empirical grounds to trace the star formation rate. This use of luminosity per unit volume reflects an attempt to decouple the stellar history of the universe from its dynamical history. Other statistical measures, such as object counts versus luminosity and redshift, are affected by the changes in the number of galactic objects via merging, as well as by evolution of stellar populations. In contrast, the change in the light budget per unit volume is only affected by the stellar production in that volume, regardless of the changes in the number of objects. Thus, for example, it makes sense to compare the total production of stars with the total metal abundance today (Cowie et al. 1988).

Popular tracers of star formation rate include the UV 1500-3000 A continuum (Connolly et al. 1997; Madau, Pozzetti, \& Dickinson 1998), the radio continuum (Mobasher et al. 1999), emission in the $\mathrm{H} \alpha$ and $\mathrm{H} \beta$ lines (Glazebrook et al. 1999), and other line emission, such as Ly $\alpha$ (Kudritzki et al. 2000) and O II (Colless et al. 1990; Hogg et al. 1998). The far-IR thermal dust emission has also been used to trace star formation rate (Hughes et al. 1998) although so far without 
the benefit of redshift information. All these measures have in common the use of some kind of luminosity per unit volume whose change is proportional to the star formation rate (with corrections). The debate over the $z>1$ slope reflects the uncertainty in the dust correction to the UV continuum measurements, which are the easiest to measure at high redshift but the most affected by dust. The other indicators are harder to measure and are affected by small-number statistics.

In this paper, we present new constraints on the history of star formation, based on the ensemble stellar populations of relatively nearby present-day galaxies $(z<0.3)$. The concept is to use the average spectrum of nearby galaxies to constrain the earlier history of star formation leading up to that stellar population. The average spectrum contains absorption features for stars of all ages and probes lookback times of $0.2-10 \mathrm{Gyr}$. With the advent of the large galaxy redshift surveys, such as the Two-Degree Field Galaxy Redshift Survey (2dFGRS) and the Sloan Digital Sky Survey main galaxy sample (SDSS mgs), it has become feasible to combine the spectra of $10^{4}-10^{5}$ galaxies to form very high signal-to-noise ratio intermediate-resolution spectra that represent the average emission of the universe at various redshifts $(0.03 \lesssim z \lesssim 0.25)$. Effectively, the surveys can be regarded as having a series of apertures on the cosmic background emission, rather than apertures on individual galaxies.

What is novel about this method is that it is not based on any luminosity output with time of the universe, unlike all the other indicators discussed above. The method uses an integral over the star formation history, rather than attempting to track the derivative (the SFR), and uses the whole visible spectrum at intermediate resolution. Perhaps the nearest approach to this in the past has been the work of Abraham et al. (1999), who used the color distribution of $z \sim 0.5$ galaxies to derive their star formation histories, which were then combined to form a cosmic star formation history. Recently, Hopkins, Irwin, \& Connolly (2001) estimated the global SFR density from star formation history measurements of the Local Group. The problem here is that the Local Group SFR may not represent the cosmic mean and is subject to large cosmic variations, such as recent "minibursts" of star formation in the Milky Way.

An advantage of this kind of "fossil cosmology" approach over the direct measurement is a reduced sensitivity to extinction. Young stars are born in dusty regions; this plagues the direct measurement approach. When they age they migrate out of such regions and contribute to the older stellar populations, we observe.

In this paper, we describe the application of this method to 166,000 spectra in the redshift range $0.03-0.25$ from the 2dFGRS (Colless et al. 2001). The plan of this paper is as follows. In $\S 2$, we describe the $2 \mathrm{dFGRS}$ data and our methods for combining the spectra. In $\S 3$, we describe our analytic models for cosmic star formation scenarios. In $\S 4$, we describe our fitting procedure and the best-fit models. In $\S 5$, we discuss the impact of possible biases on our results from aperture effects and luminosity selection. Finally, in $\S 6$ we give our conclusions.

\section{THE 2dFGRS DATA}

The 2dF Galaxy Redshift Survey is a magnitude-limited spectroscopic survey (Maddox et al. 1998; Colless et al. 1999; Colless et al. 2001) using the Anglo-Australian Observatory's $2 \mathrm{dF}$ facility, which is capable of observing up to 400 galaxies simultaneously (Taylor \& Gray 1990; Lewis, Glazebrook, \& Taylor 1998; Lewis et al. 2002). The magnitude limit of the survey is an extinction-corrected $b_{J}$ of 19.45 selected from the Automated Plate Measuring (APM) galaxy catalog (Maddox, Efstathiou, \& Sutherland 1990a, 1996; Maddox et al. 1990b). By the end of the 2dFGRS survey (2002 January), up to 250,000 unique galaxy redshifts are expected to have been measured. The survey covers approximately $2000 \mathrm{deg}^{2}$ of sky distributed between the north and south Galactic poles (NGP and SGP) in high Galactic latitude fields. A full description of the survey geometry is given by Colless et al. (2001).

By 2001 June, about 173,000 unique galaxy redshifts had been measured. This is the sample used in the analysis presented in this paper. The spectra are observed through a fixed 2". 1 fiber aperture, and the wavelength coverage varies only slightly from observing run to run, consistently covering the range $3700-7860 \AA$ with a 2.1 pixel resolution FWHM of $9.0 \AA$. All the spectra in the survey have been eyeballed and assigned a quality $Q$ from 1 to 5 (Colless et al. 2001): 1 , no identifiable redshift; 2 , a possible redshift; 3 , a $90 \%$ reliable redshift; 4, a 99\% reliable redshift; and 5, a 99\% reliable redshift with a high-quality spectrum. The survey is considered to consist of those galaxy spectra with $Q \geq 3$ (approximately $92 \%$ of galaxies observed). These galaxies have a median redshift of about 0.11 , and the typical spectral signal-to-noise ratio at the survey limit is about 10 per pixel.

We construct our "cosmic spectra" in redshift slices $z \rightarrow z+\Delta z$ by applying an instrument response correction, deredshifting to the rest frame, and summing up all the $Q \geq 3$ spectra in the interval $\Delta z$. The galaxies are scaled to match their measured $b_{J}$ luminosity by comparison with an integration of the $b_{J}$ filter curve over the measured spectrum (in the observed frame). The scaling allows for the fact that the fibers sample only a fraction of a galaxy's light and for extinction and exposure-time variations between observations. A maximum scaling is allowed (as a function of apparent magnitude) to avoid adding excessive noise from poorquality spectra. This scaling limit is only applied to $5 \%$ of the data. Finally, the cosmic spectrum is normalized to a mean of unity over a set wavelength range; to reiterate, our method of analysis uses spectral features, not absolute luminosities, other than for the weighting of the galaxy spectra. This spectrum represents the spectral emission per unit volume in the $z \rightarrow z+\Delta z$ interval, down to the limiting magnitude of the survey. As a result of the $b_{J} \approx 19.45$ limit of the 2dFGRS, the corresponding limiting absolute luminosity will be higher at higher redshifts. This exclusion of lower luminosity galaxies is a possible source of bias. This is quantified in $\S 5$.

The instrument response correction consists of applying the average $2 \mathrm{dF}$ spectral response, as given by Lewis et al. (2002). Only a simple scaling is applied for light lost outside the fixed angular size fiber aperture which is another potential source of bias to be discussed in $\S 5$.

Examples of cosmic spectra, at a series of redshifts, are shown in Figure 1. As might be expected from a broad star formation history, the average emission from the universe looks remarkably like an $\mathrm{Sb}-\mathrm{Sbc}$ galaxy spectrum (Kennicutt 1992). 


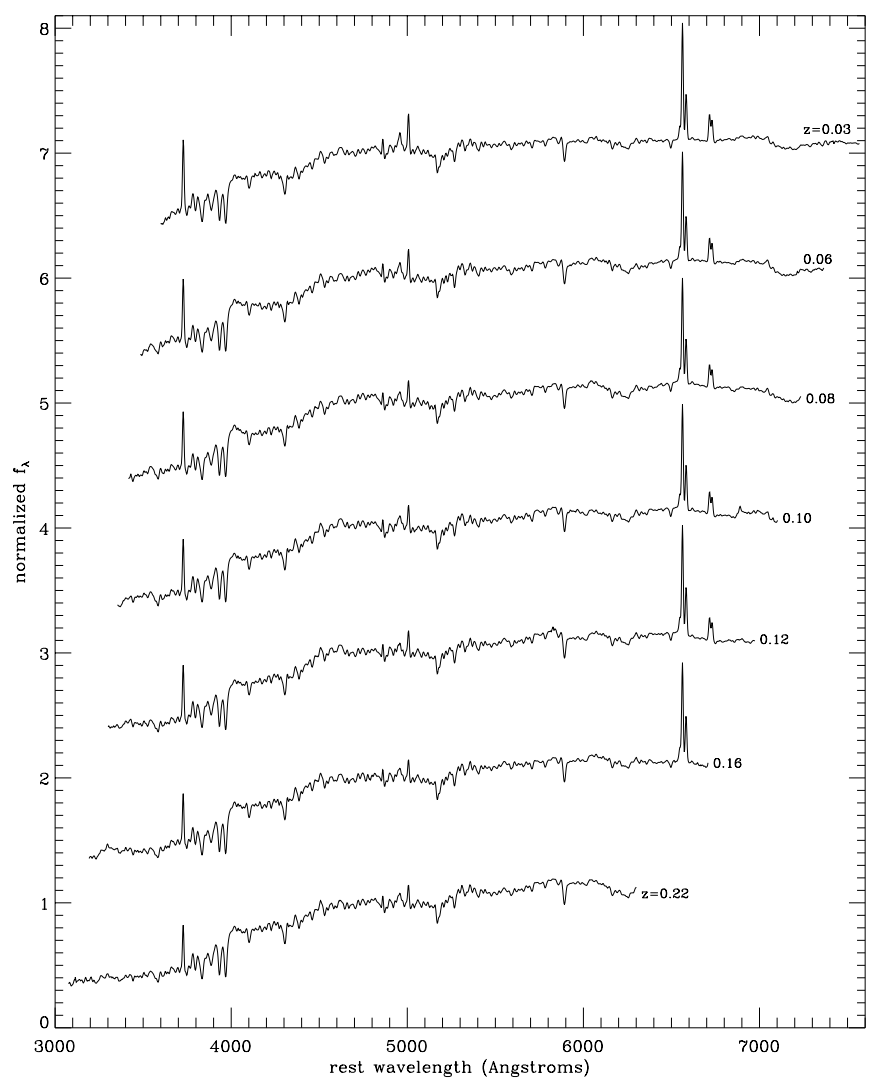

FIG. 1.-Averaged 2dFGRS spectra from various redshift bins $(0.025-$ $0.04,0.06-0.07,0.08-0.09,0.10-0.11,0.12-0.13,0.155-0.175,0.20-0.25)$. The spectra were normalized to a mean of 1.0 between 4200 and $5800 \AA$ and offset by 1.0 from each other.

\section{COSMIC STAR FORMATION SCENARIOS}

First we must decide how best to parameterize cosmic star formation histories. We use two different families of parameterization. The first is a physically motivated parameterization, using an infall model to describe star formation as a function of time and timescales. The second is a purely empirical model, which describes star formation as a series of power laws with redshift. This facilitates direct comparisons with the literature.

For the cosmology we consider two types, both constrained to be flat in accord with the cosmic microwave background data (de Bernardis et al. 2000):

1. The emerging standard cosmology (which we denote $\mathscr{C} 1$ ), which has cosmological parameters $C_{0}=\left(h, \Omega_{m_{0}}, \Omega_{\Lambda_{0}}\right)=(0.70,0.3,0.7) .{ }^{14}$ These parameters are the current best constraints from a variety of observations (Silk 1999).

2. A longer age cosmology (which we denote $\mathscr{C} 2$ ) with $C_{0}=(0.55,0.2,0.8)$. These parameters have been adjusted on the longer age side to the margins of consistency with modern limits.

For galaxies at $z=0.1$ with $z_{\text {form }}=5$, these cosmologies give ages of 11.0 and $15.7 \mathrm{Gyr}$, respectively.

Given a star formation history and a cosmology, we can compute spectra using standard evolutionary synthesis codes. We used the PEGASE code (Fioc \& Rocca-
Volmerange 1997). ${ }^{15}$ Both parameterizations require an initial mass function (IMF), and we choose the Salpeter (1955) power-law slope, with stellar mass in the range $0.1-120 M_{\odot}$. We have also investigated the Kennicutt (1983) IMF over the same mass range. We also have choices to make on metallicity evolution and dust extinction. For the former, the enrichment of the interstellar medium is determined by using the calculations of Woosley \& Weaver (1995) within the PEGASE code. For the dust extinction, the prescription for an inclination-averaged disk geometry is used. However, the choice of extinction, $E(B-V) \sim 0.2 \pm 0.1$, makes negligible difference to the results in this paper for two reasons: (1) we are only determining the relative SFR as a function of time or redshift, i.e., not comparing predicted luminosity with luminosity density; and (2) the robust results are primarily constrained by using high-pass-filtered spectra, which are insensitive to the extinction model. We note that there will be a second-order effect from any systematic variation of extinction with spectral type and/or luminosity.

\subsection{Physical Parameterization}

The first parameterization is a natural scenario. Star formation starts at $z=z_{\text {form }}$. Gas falls into sufficiently dense regions to form stars with an infall timescale $t_{i}$ :

$$
M_{\text {galaxy }}=1-e^{-t / t_{i}} .
$$

The gas in these regions forms stars at a rate $(\mathscr{P})$ proportional to the amount of gas available with a star formation timescale $t_{s}$ :

$$
\mathscr{S}=M_{\mathrm{gas}} / t_{s} .
$$

This parameterization is readily implemented in the PEGASE population synthesis code (Fioc \& RoccaVolmerange 1997). The code includes recycling of ejecta into the interstellar medium (ISM) and consistent evolution of the metallicity. An analytical approximation to the star formation rate can be determined, assuming an instantaneous-recycling approximation ( $f$ is the mass fraction of stars that are not returned to the ISM). Under this prescription, the star formation rate evolves as

$$
\frac{\mathrm{d} \mathscr{S}}{\mathrm{d} t}=\frac{1}{t_{s}}\left(-f \mathscr{S}+\frac{e^{-t / t_{i}}}{t_{i}}\right) .
$$

For $f t_{i} \neq t_{s}$, the solution for the star formation rate with time is given by

$$
\mathscr{S}=\frac{e^{-t / t_{i}}-e^{-f t / t_{s}}}{f t_{i}-t_{s}},
$$

with initial condition $\mathscr{S}=0$ at $t=0$. The normalization is such that the total mass of gas available is unity. This parameterization is useful, in that it is physically motivated and that it allows for a rise at early times and a fall at late times of the universal star formation rate. The deviation between the approximation and the code becomes apparent when recycling from lower mass stars becomes significant. An example is shown in Figure 2, with $f=0.7$ and 0.8 for the recycling approximations.

\footnotetext{
${ }^{15}$ PEGASE Version 2, revised 2001 May 5, is available at http:// www.iap.fr/users/fioc/PEGASE.html.
} 


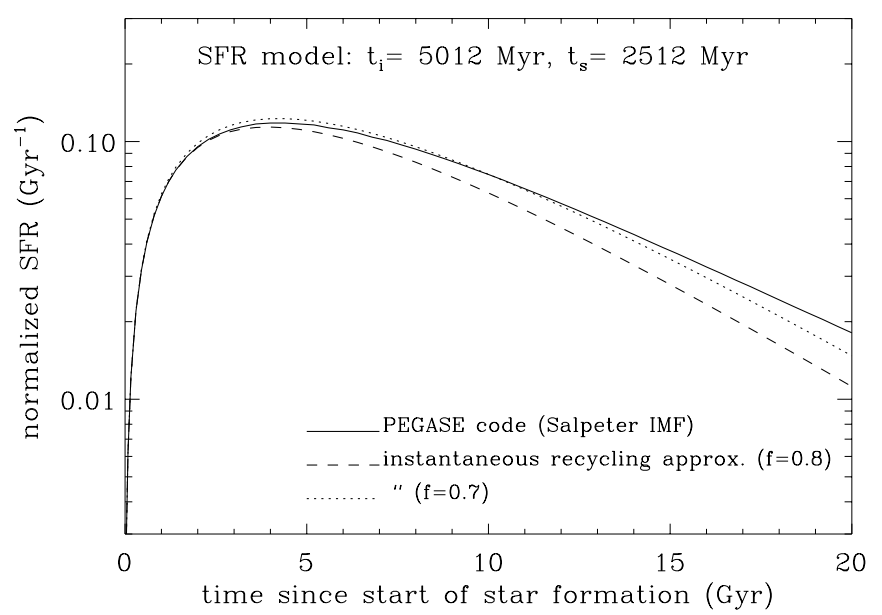

FIG. 2.-Example star formation scenario using an infall timescale and a star formation timescale. The instantaneous-recycling approximation, eq. (4), assumes that a mass fraction $R(=1-f)$ of stars formed are returned instantaneously to the ISM. The normalized SFR is relative to the total mass of gas available for forming stars. The integral of the SFR can be greater than unity because of recycling of material back to the ISM.

\subsection{Empirical Parameterization}

In the second parameterization, we empirically make the star formation rate a function of redshift. In particular, we make the star formation rate between $z=5$ (our choice of formation redshift) and $z=1$ proportional to $(1+z)^{\alpha}$ and that between $z=1$ and $z=0$ proportional to $(1+z)^{\beta}$, with matched SFR at $z=1$. This form allows us to make a comparison of the best-fitting parameters with previous studies that measure star formation rate with redshift via luminosity densities. In particular we can compare the value of $\beta$ defined and measured by Hogg (2001), as well as other measurements of the variation of the comoving SFR density, with redshift (see, e.g., Lilly et al. 1996; Madau et al. 1996).

The total amount of star formation $(r)$ between $z=5$ and $z=0$, as a fraction of the mass available, was normalized to a range of values from $r=0.3$ to $r=1.4$ (the SFR normalization). In other words, the mass of gas available for star formation is unity and the $r$ is the total mass of stars formed since $z_{\text {form. }}$. This can be greater than unity because of recycling of material back into the interstellar medium. Higher $r$-values result in higher average metallicity, because the fusion products released into the ISM by supernovae are more abundant relative to the remaining gas.

\section{BEST-FIT STAR FORMATION SCENARIOS}

\subsection{Reductions}

The 2dFGRS data were divided into 14 redshift bins between $z=0.025$ and $z=0.25$, each containing about 12,000 spectra. Half the redshift bins were below $z=0.11$ (the median redshift of the survey). The lower and higher groups of seven redshift bins were considered separately and together to test the robustness to varying aperture and selection effects (see $\S 5$ for a further discussion of this).

For each redshift bin, the spectra were divided into 10 positional bins based on their coordinates (four regions in the NGP and six in the SGP). A normalized average spec- trum was calculated and the positional bins were used to estimate the uncertainties. This spectrum represents the total optical emission of all galaxies in the volume of the redshift shell down to the 2dFGRS magnitude limit. The absolute magnitude limit is fainter than or about $M^{*}$ out to a redshift of 0.2 (the depth is discussed further in $\S 5$ ).

For flux-calibration the spectra were divided by the $2 \mathrm{dF}$ response function of Lewis et al. (2002) before the fluxes were co-added at their rest wavelengths. Spectra contribute to the final spectrum in proportion to their $b_{J}$ luminosity. In addition, the averaged spectra were smoothed and resampled to match approximately the much lower $20 \AA$ resolution of the spectral library (Lejeune, Cuisinier, \& Buser 1997) used by the population synthesis code.

\subsection{Goodness of Fit}

To evaluate the goodness of fit between a star formation scenario and the 2dFGRS data, we compare the spectrum from each redshift bin with the appropriate model spectrum (at the same age) from the scenario. Fiber spectra are known to be difficult to flux accurately, so it is desirable to develop methods that are insensitive to small spectrophotometric uncertainties.

The first method we used was to allow for the possibility of spectrophotometric calibration errors by including a correction function before evaluating the fit. This was to account for spectrophotometric discrepancies between the $2 \mathrm{dF}$ response curve used to calculate the average spectra and the true average $2 \mathrm{dFGRS}$ response. We used a fourthorder polynomial with observed wavelength for this spectrophotometric correction, the coefficients being determined by ratioing the model and data spectra (excluding the strongest emission lines, as per the fitting). An important point is that the correction is constrained to be the same function at all redshifts; thus, the degeneracy between the spectrophotometric correction and the model fitting is partly broken, because of the range of redshift covered (0.03-0.25). Typically, in our fitting we find this spectrophotometric correction to be of the order of a $5 \%-10 \%$ change in the response function of Lewis et al. (2002) for the best-fitting models (within $3 \sigma$ ). These values represent the rms relative difference over the wavelength range between the polynomial correction and a constant value (representing changes in the relative, not absolute, spectrophotometry). Because the response function was measured from standard stars observed during a single hour, such a difference could arise from (1) unaccounted-for reduction or throughput discrepancies between extended and point sources and /or (2) variations in the instrument response over the 2dFGRS survey time.

In evaluating the fit between a model spectrum and a 2dFGRS data spectrum, we compare the low-pass and high-pass information separately to form two figures of merit (FOMs) from the normalized spectra. This is illustrated in Figure 3. The normalization is to set a mean of unity over the rest-wavelength range 4100-6200 $\AA$, with the lower limit being just above the $4000 \AA$ break and the upper limit being just in the detected range for galaxies with $z=0.25$. Both the normalized model spectrum and the normalized data spectrum are smoothed by using a top-hat function of width $200 \AA$ (10 sampling points). The original spectra are divided by the smoothed spectra to produce the high-pass spectra. FOM A is the reduced $\chi^{2}$-value from the 

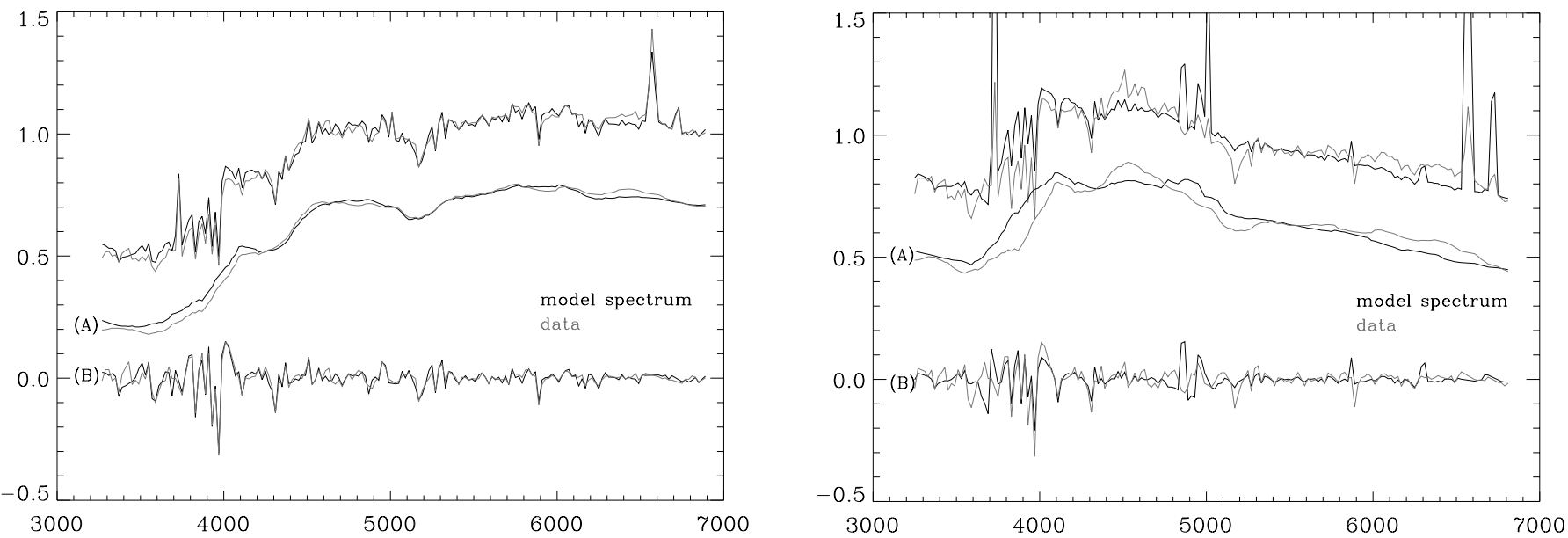

Fig. 3.-Example model and data spectra, with the low-pass (A) and high-pass (B) information separated. The data have been adjusted for possible spectrophotometric errors, as described in $\S 4.2$. FOM A is the reduced $\chi^{2}$-value determined from the difference between the smoothed spectra (offset by -0.3 in the plot). FOM B is determined from the high-pass spectra (offset by -1.0 ). Note that the strongest emission lines were excluded from the fitting procedure. The left plot shows a good fit with reduced $\chi^{2}$-values of order unity for both figures of merit. The right plot shows a poor fit for both FOMs $\left(\chi^{2} / \nu \sim 17\right.$ and 6 for $\mathrm{A}$ and $\mathrm{B}$, respectively). [See the electronic edition of the Journal for a color version of this figure.]

difference between the low-pass spectra and FOM B is the reduced $\chi^{2}$-value from the high-pass spectra.

This high-pass FOM B is thus robust against any largescale errors in the spectrophotometry, and in fact we find that FOM B is negligibly different whether we include a spectrophotometric correction or not. FOM B places more reliable constraints on the best-fit star formation scenarios, since it is less affected by the systematic uncertainties of spectrophotometry and extinction. FOM A is an independent check: our aim is to obtain consistent histories from both the low-pass (continuum) and high-pass (absorption line) information. From the best-fit star formation we can obtain a range of probable spectrophotometric corrections that were used to determine the mean and uncertainty in the $b_{J} k$-corrections of the galaxy spectra (Madgwick et al. 2002).

In order to determine the uncertainties in our set of spectra we take the empirical approach of dividing up the survey into 10 separate, approximately equal sky areas. We compute the mean spectrum separately for each area and use the variance between them to set out errors as a function of wavelength. The rms errors computed this way are around $0.2 \%-1 \%$ for the high-pass spectrum and $1 \%-3 \%$ for the low-pass spectrum. An additional uncertainty of $1.5 \%$ per wavelength bin is added in quadrature to account for intrinsic model inaccuracies due to, for example, the signal-tonoise ratio of the spectra in the input atlas. This uncertainty value was chosen so that the reduced $\chi^{2}$-values for the bestfit star formation scenarios were approximately unity.

For a given star formation scenario, we average the FOM values over all the redshift bins. We calculate the figure-ofmerit quantities by summing over all wavelengths except near strong nebular emission lines (O II $\lambda 3727$; $\mathrm{O}$ III $\lambda 5007$; $\mathrm{H} \alpha \lambda 6563 ; \mathrm{N}$ II $\lambda 6583$; $\mathrm{S}$ II $\lambda 6716, \lambda 6730)$. We do this because our goal is to calculate the star formation history from the stellar emission only. The nebular line emission is a measure of the instantaneous star formation rate in $\mathrm{H}$ II regions, but the PEGASE code uses oversimple assumptions to translate star formation rate to a set of line ratios. Since understanding ionization levels and interpreting line ratios are complex subjects, and since the current strength of emission lines does not constrain past star formation rates, we choose not to use this information. In addition, the emission lines will be contaminated by active galactic nuclei in a few percent of our galaxies, so ignoring the lines will minimize the bias this causes.

\subsection{Results}

For the fitting procedure we calculate a grid of model values, $\left(t_{i}, t_{s}, z_{\text {form }}\right)$ for the physical parameterization and $(\alpha, \beta$, $r$ ) for the empirical parameterization. We estimate that after our data processing there are approximately 20 degrees of freedom for FOM A (about 22 independent wavelength points) and 200 degrees of freedom for FOM B. The minimum $\chi^{2} / \nu$-value was about unity for both figures of merit, based on the uncertainties described above. We believe the additional uncertainty of $1.5 \%$ necessary to obtain a suitable $\chi^{2}$-value reflects the remaining imprecision of the model spectra, because these types of models were conceived originally to model galaxy colors. Improved, higher resolution models will be required in future work. We proceed to interpret the star formation laws of the best-fitting models. We note that the best fit matches the data to about $2 \% \mathrm{rms}$, so in absolute terms we are accounting for the volumetric light emission very well. The problem remains that the high-pass data are much better than the current state of the art in population synthesis modeling.

In order to estimate the confidence limits on the FOMs $\left(\Delta \chi^{2} / \nu\right)$, we investigated the variation of the best-fit parameters, using Monte Carlo simulations. It is not sufficient to apply the standard confidence limits because of correlated uncertainties between different wavelength points. The investigation included using the spectra from the 10 positional bins separately, applying random errors of $1.5 \%$, using different redshift ranges (e.g., 0.03-0.11, 0.11-0.25), and varying the dust extinction in the models. As expected, FOM B is fairly robust, and we use $\Delta \chi^{2} / \nu=0.15$ for the $3 \sigma$ $(99.73 \%)$ confidence limit. FOM A is strongly affected by using different positions on the sky and different redshift 

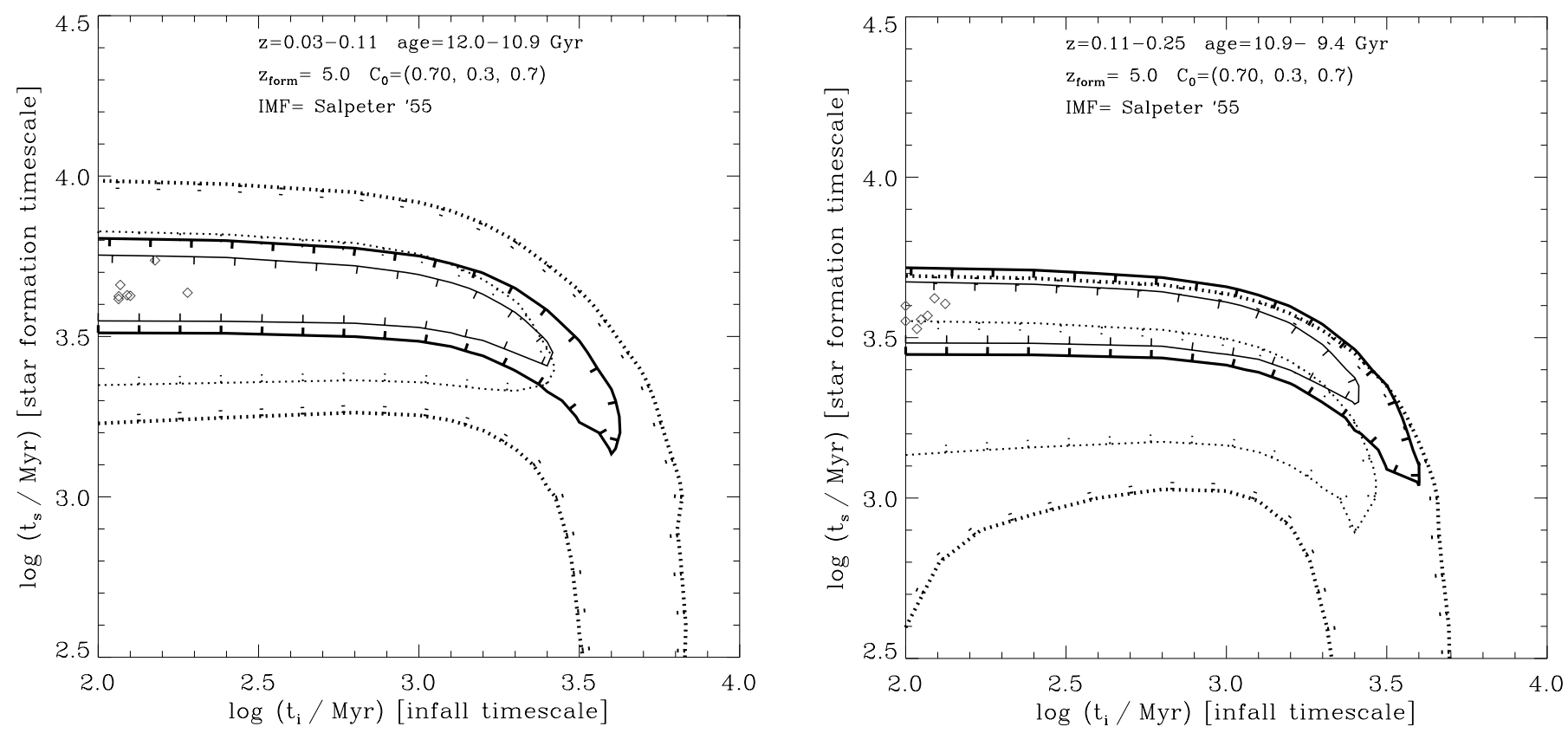

FIG. 4.-Best-fit regions of $\log t_{s}$ vs. $\log t_{i}$ for cosmology $\mathscr{C} 1$ with $z_{\text {form }}=5$. The two plots use different redshift ranges from the data. The contours represent the $2 \sigma$ and $3 \sigma$ formal confidence limits, with dotted lines for FOM A and solid lines for FOM B. The FOMs were calculated using the average from seven redshift bins at each grid point. The diamonds represent the FOM B best-fit parameters for each bin. Section 3.1 describes the cosmic SFR parameterization using a star formation timescale $\left(t_{s}\right)$ and an infall timescale $\left(t_{i}\right)$. [See the electronic edition of the Journal for a color version of this figure.]

ranges. This is principally a result of spectrophotometric uncertainties, and we use $\Delta \chi^{2} / \nu=2.1$ for the $3 \sigma$ limit (when plotting contours using the full redshift range). In neither case is the FOM significantly affected by changes in the dust extinction used in the model. This is not surprising for FOM B, since it is determined from high-pass spectra. A spectrophotometric adjustment is applied to the data before determining the FOMs, and therefore FOM A is principally sensitive to variations over ranges of $200 \AA$ (the smoothing length), such as the $4000 \AA$ break, and is less sensitive to extinction.

The best-fit parameters are fairly degenerate. Figure 4 shows the best-fit regions in $\log t_{s}$ versus $\log t_{i}$ space with $z_{\text {form }}=5$. The results for the lower and higher redshift ranges are shown separately. The solid contours represent the $95.4 \%$ and $99.73 \%(2 \sigma$ and $3 \sigma)$ confidence boundaries for FOM B, while the dotted contours are for FOM A. Notably, the regions contained by the FOM B boundaries at low and high redshift are similar, whereas the FOM A boundaries are significantly different. We do not overinterpret this, noting that FOM A is less reliable because of spectrophotometric uncertainties. The best-fit model (FOM B) has $t_{s} \sim 4000 \mathrm{Myr}$ with $t_{i} \lesssim 200 \mathrm{Myr}$ (note degeneracy for low $t_{i}$ ). Both redshift ranges have $t_{i} \lesssim t_{s}$ within the $2 \sigma$ levels, even though the SFR is similar on interchange of $f t_{i}$ and $t_{s}$ (see approximation, eq. [4]). However, the metallicity evolution is different, depending on whether infall of new gas or the star formation timescale determines the SFR at late times.

The figure-of-merit values become degenerate for $t_{i} \ll t_{s}$ (galaxies form quickly in comparison with the star formation timescale), while maintaining a good fit. In Figure 5, $t_{i}$ is set at $100 \mathrm{Myr}$, and the best-fit regions of $\log t_{s}$ versus $\log$ $z_{\text {form }}$ are identified for cosmologies $\mathscr{C} 1$ and $\mathscr{C} 2$. At the $3 \sigma$ limits (FOM A and $\mathrm{B}$ ), the redshift of formation is greater than or about 0.65 .

To aid understanding of the meaning of our results, Figure 6 shows a variety of star formation histories that are within the $3 \sigma$ limits. The first plot shows scenarios with $t_{i} \ll t_{s}$, i.e., those identified from Figure $5(\mathscr{C} 1)$. The second plot (Fig. 6) shows "smoother" scenarios with $t_{i} \sim t_{s}$, i.e., those identified from plots similar to those shown in Figure 4, the right edges of the $3 \sigma$ boundaries.

The best-fit scenarios cover a large range of $z_{\text {form }}$ but have the following in common: the normalized SFR at $z=0$ is in the range $0.02-0.04 \mathrm{Gyr}^{-1}$, and $84 \%-92 \%$ of stars formed before $z=0.3$ for $\mathscr{C} 1$.

It can be seen that the main constraint is on the slope of the star formation rate for $0<z<1$. Fitting an SFR of $(1+z)^{\beta}$ from $z=0$ to 1 to the natural scenarios with $z_{\text {form }}>1$, we obtain from the $3 \sigma$ confidence limits $1 \lesssim \beta \lesssim 4.5(\mathscr{C} 1)$ and $1 \lesssim \beta \lesssim 4(\mathscr{C} 2)$. If we fit an SFR of $(1+z)^{\alpha}$ from $z=1$ to 4 , there is no lower limit on $\alpha$, since at least one of the well-fitting models has little or no star formation before $z=1$. The upper limit from these natural scenarios is $\alpha \lesssim 1$ for both cosmologies.

The $t_{i}-t_{s}-z_{\text {form }}$ parameterization is limited in its scope for changes of SFR with time. Therefore, to further test star formation history, we look at the $\alpha-\beta-r$ parameterization. Figure 7 shows best-fit regions in $\alpha$ versus $\beta$, with $r=1.1$ for both cosmologies.

In these scenarios, the galaxies start fully constituted with gas (there is no infall) and consistent evolution of the metallicity is implemented. Constant-metallicity scenarios were also tested but found not to be consistent within the $3 \sigma$ limit on FOM B. If we take an SFR normalization of 1.1, meaning a total mass of stars formed between $z=5$ and $z=0$ equal to 1.1 times the mass of gas available, there is a degen- 

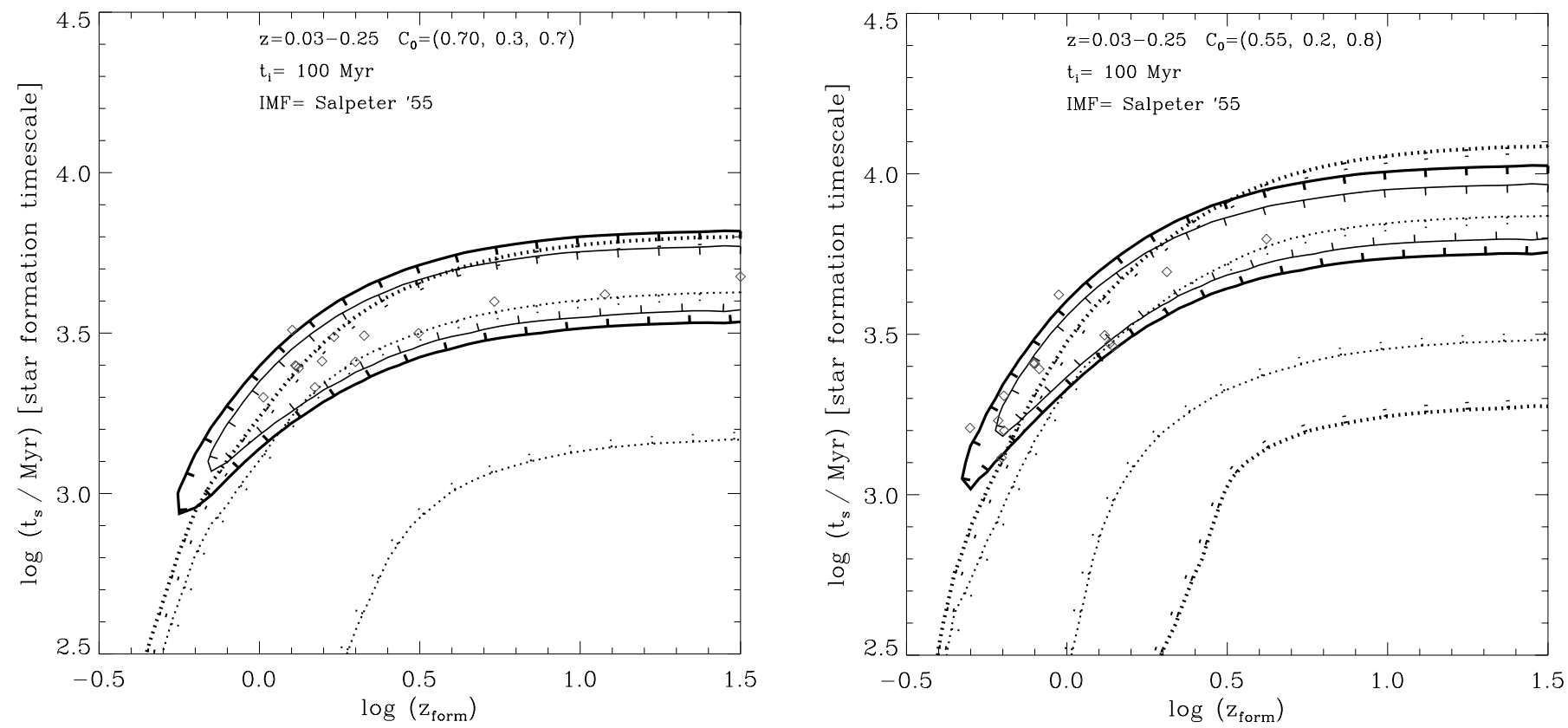

Fig. 5. - Best-fit regions of $\log t_{s}$ vs. $\log z_{\text {form }}$ for two different cosmologies. Contours as in Fig. 4. [See the electronic edition of the Journal for a color version of this figure.]

eracy across the plane of $\alpha$ versus $\beta$ (Fig. 7). Scenarios with $\beta<0$ cannot be ruled out for $\alpha>2.5$. However, this would imply a minimum in the SFR around $z=1$, which is in disagreement with many cosmic SFR density studies based on photometry. These also represent a branch of solutions that are impossible to produce using the physical parameterization. The conservative upper limit of this study is $\beta<5$. Taking the conservative lower limit on $\beta$ from the analysis by Hogg (2001), $\beta>1.3$, we obtain $\alpha<3$ (for $\mathscr{C} 1$ ). If we assume $\alpha \lesssim 0$ (i.e., star formation declined or remained constant for $z>1$, which corresponds to the lower right branch of solutions in Fig. 7), then we obtain a range of $1.5<\beta<5$. This is in very good agreement with Hogg's results, which represent an ensemble of different luminosity density-based indicators.

Figure 8 shows best-fit regions in $\alpha$ versus $r$, with $\beta=3$, for $\mathscr{C} 1$. The two plots show the results for different IMFs.
There is no lower limit on $\alpha$, given $\beta=3$. There are upper limits of $\alpha<0.5$ for the Salpeter (1955) IMF and $\alpha \lesssim 1$ for Kennicutt (1983) IMF, with $\alpha<0$ at the $2 \sigma$ confidence level for both IMFs. There is some degeneracy between the final metallicity $(z=0)$ and the IMF. For example, if we take star formation scenarios within $\alpha>-1$ and the $3 \sigma$ confidence boundaries, there are scenarios with around solar to twice-solar metallicity using the Salpeter IMF and with around half-solar to solar metallicity using the Kennicutt IMF.

With $\beta \gtrsim 3$, the best-fit models have a decrease in SFR before $z=1$ ( $2 \sigma$ limit of FOM B). Figure 9 shows best-fit regions in $\alpha$ versus $r$, with $\beta=2$, for $\mathscr{C} 1$. Here, the best-fit models are consistent with a plateau in SFR before $z=1$ or a marginal increase or decrease. An upper limit would be $\alpha<1.5(2 \sigma)$, if $\beta>2$, as is suggested by most direct methods of tracing the cosmic SFR (Hogg 2001).
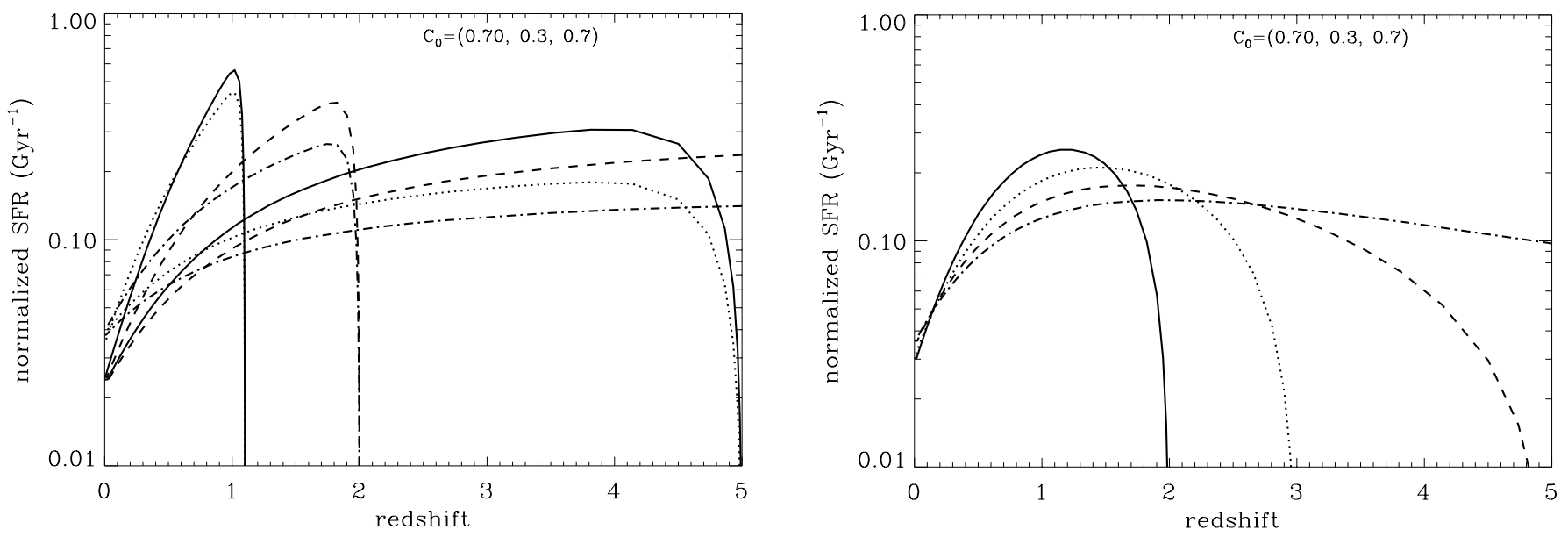

Fig. 6.-Example set of star formation scenarios that are within $3 \sigma$ of the minimum FOM values for $\operatorname{cosmology} \mathscr{C} 1$. Left: Scenarios with $t_{i} \ll t_{s}$; right: scenarios with $t_{i} \sim t_{s}$. [See the electronic edition of the Journal for a color version of this figure.] 

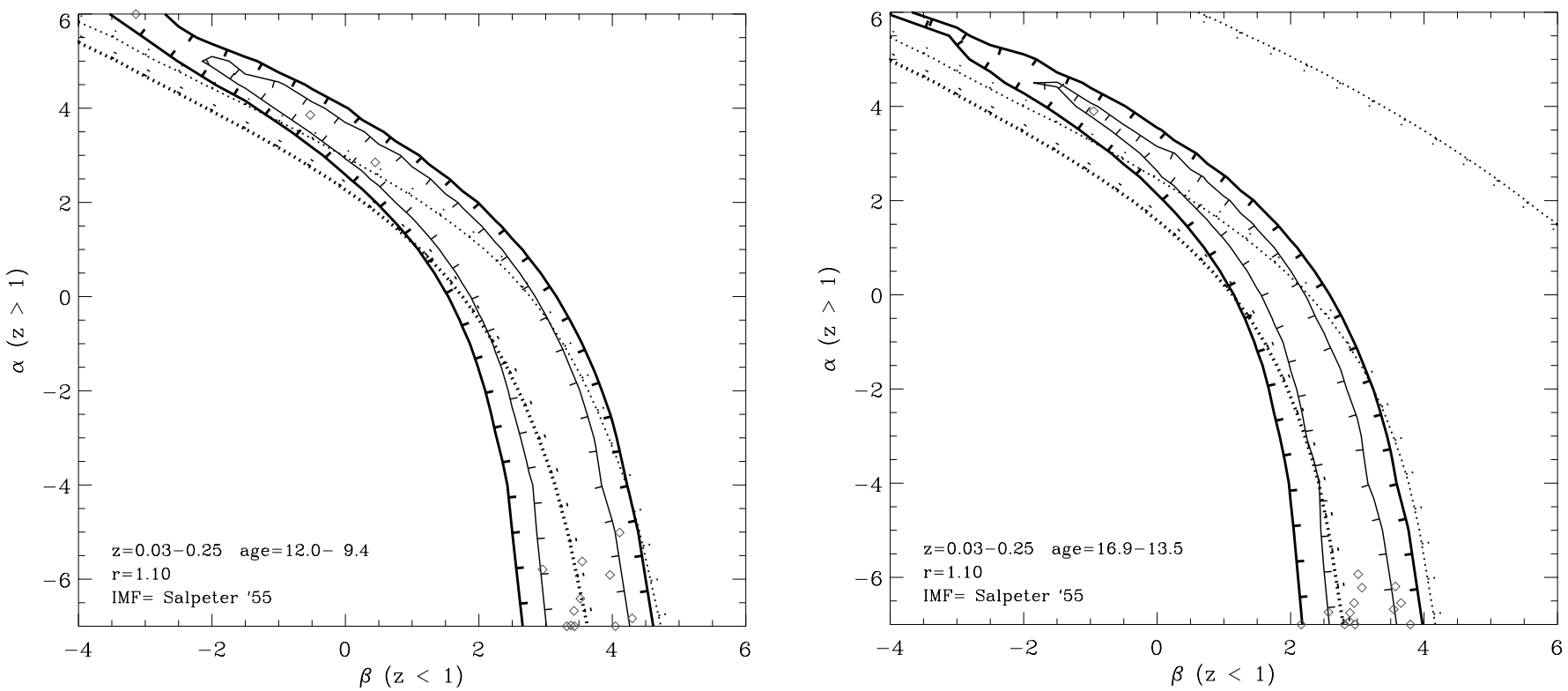

Fig. 7.-Best-fit regions of $\alpha$ vs. $\beta$ for cosmologies $\mathscr{C} 1$ (left) and $\mathscr{C} 2$ (right). Contours as in Fig. 4. Section 3.2 describes the cosmic SFR parameterization, with $\mathscr{S} \propto(1+z)^{\beta}$ for $0<z<1$ and $\mathscr{S} \propto(1+z)^{\alpha}$ for $1<z<5$. [See the electronic edition of the Journal for a color version of this figure.]

\section{POSSIBLE SELECTION EFFECTS AND BIASES}

All spectroscopic information is subject to the effect of aperture bias (see, e.g., the discussion of Kochanek, Pahre, \& Falco 2000). In particular, the aperture may be too small to encompass a representative fraction of a galaxies light. This effect will obviously be greater at lower redshift.

Figure 10 shows the increase in the effective size of a $2 \mathrm{dF}$ 2 ". 1 fiber aperture with redshift. At $z=0.1$ this is $2.7 h^{-1}$ $\mathrm{kpc}$, which is comparable to a typical large disk scale length of $3 h^{-1} \mathrm{kpc}$ (de Jong \& Lacey 2000). In practice, the effective aperture is more like $3.4 h^{-1} \mathrm{kpc}\left(2{ }^{\prime \prime} 6\right)$, because the median observation seeing was about 1 ". 5 . Thus, it is reason- able that the effect of aperture bias will be much smaller for $z>0.1$, because we are sampling more than half the total light of galaxies.

The data allow for a further test of this because of its own internal seeing variations. Spectra taken in bad atmospheric seeing are a rough proxy for spectra taken through a larger aperture, because the object is smeared out over a disk about the size of the seeing disk. As a test of this, we chose a sample of about 1500 galaxies $(z<0.15)$ that had measured spectra taken both in relatively good seeing $\left(\lesssim 11^{\prime \prime} 5\right)$ and in poor seeing $\left(\gtrsim 3^{\prime \prime}\right)$ such that the difference in representative aperture was greater than a linear factor of 1.5 for each galaxy with $Q \geq 3$ for both spectra. With this sample, we mea-
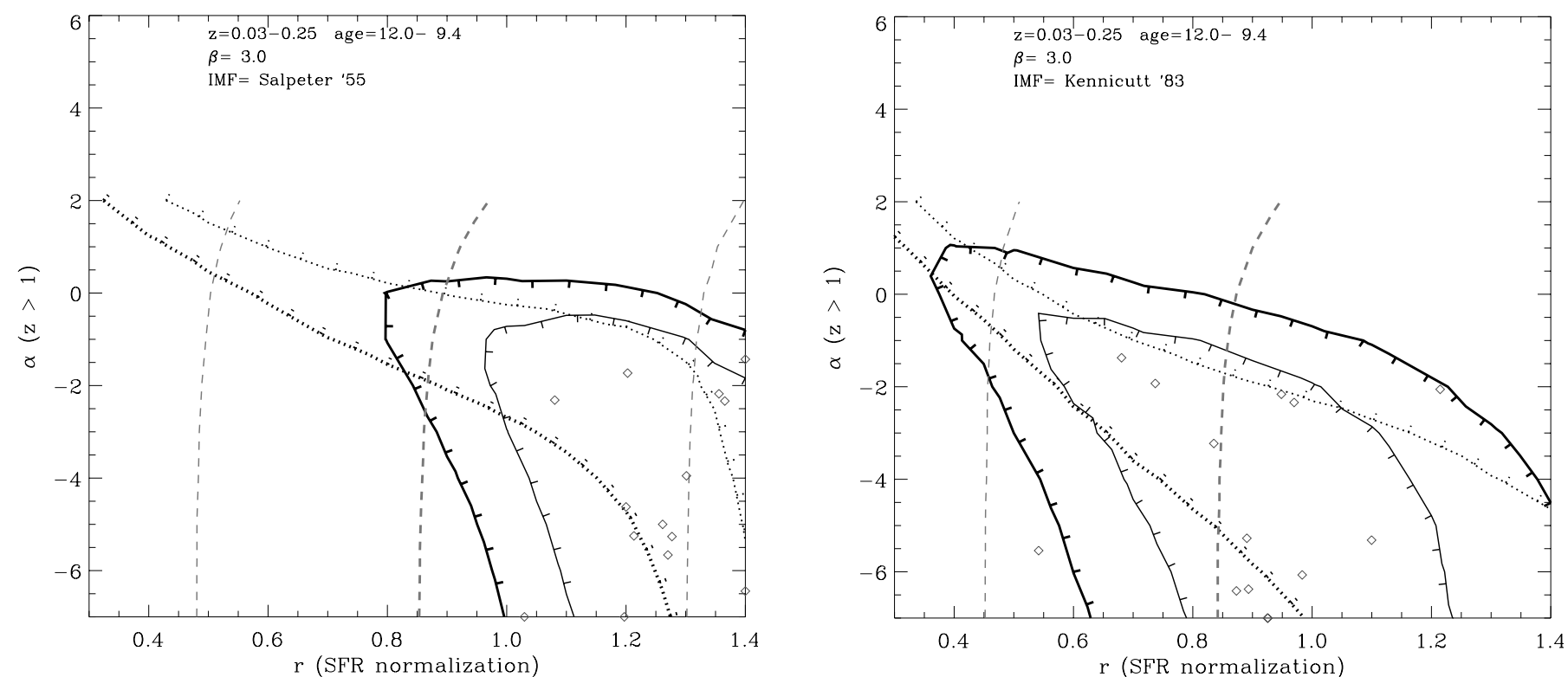

FIG. 8.-Best-fit regions of $\alpha$ vs. $r$, with $\beta=3$. The two plots are for different IMFs with cosmology $\mathscr{C} 1$. Contours as in Fig. 4 . In addition, the vertical dashed lines show the final metallicity, averaged on the luminosity: from left to right, $Z=0.01,0.02$, and 0.04 (left) and 0.01 and 0.02 (right). [See the electronic edition of the Journal for a color version of this figure.] 

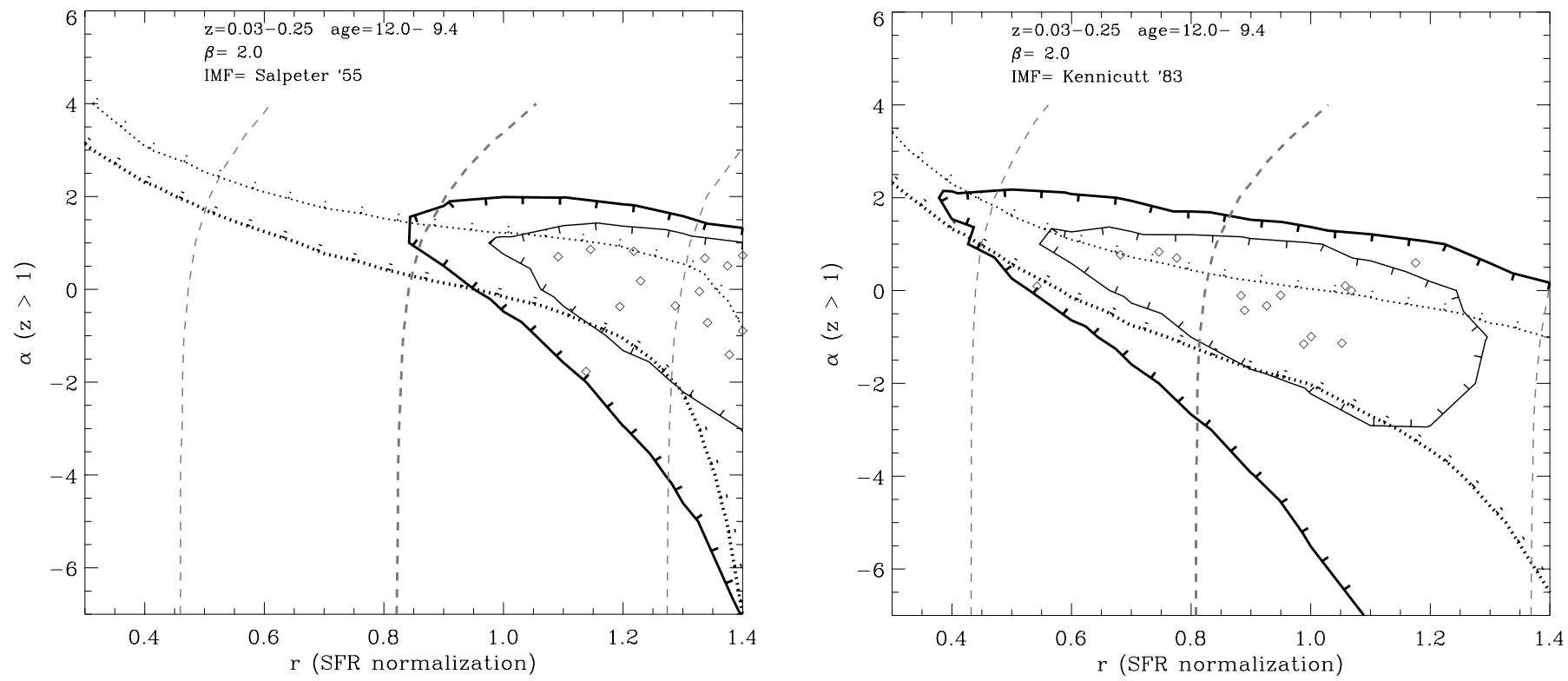

FIG. 9.-Same as Fig. 8, but for $\beta=2$. [See the electronic edition of the Journal for a color version of this figure.]

sured the change in equivalent width (EW) of a number of lines between the larger representative aperture and the smaller aperture. In some individual spectra, the sky subtraction was inadequate for accurate EW measurement. Therefore, measurements for which the reduced counts were too low or contaminated by sky-emission lines were excluded. For further robustness, the galaxies were divided into subsamples of 10 as a function of redshift, and the median change in EW of each group was determined. The results are shown in Figure 11. Similar results were obtained if a weighted mean (by counts) was used, rather than a median.

There is no evidence of any aperture effect in terms of the average change of EW for the galaxies in this sample, given a difference in representative aperture with a linear factor in the range 1.5-2.3. We cannot rule out a small aperture effect on the measured cosmic spectra, particularly for $z<0.05$, where the test sample is small.

How do the measured cosmic spectra, as a function of redshift, vary with selection effects, aperture effects, if any,
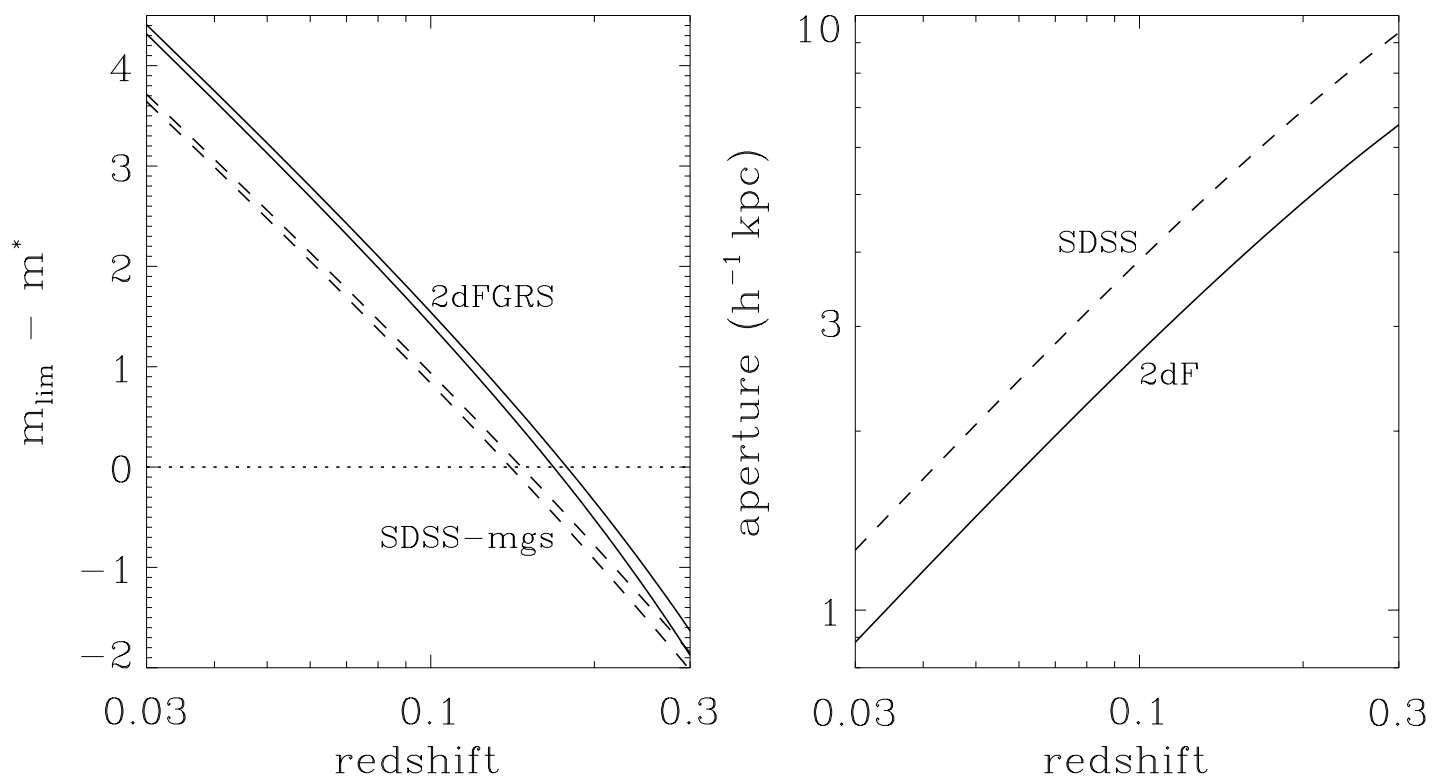

FIG. 10.- Selection and aperture effects in the 2dFGRS and, for comparison, the SDSS mgs. The left plot shows the limiting magnitude relative to the characteristic magnitude of the Schechter (1976) luminosity function vs. redshift. For the 2dFGRS $\left(b_{J}\right): m_{\lim } \approx 19.45 ; m^{*} \approx-19.79+5 \log D_{L}+1.9 z+2.7 z^{2}$ (Madgwick et al. 2002); the solid lines show the limits, with \pm 0.04 error in $M^{*}$ and $\pm 10 \%$ error in the $k$-correction. For the SDSS $\left(r^{\prime}\right): m_{\mathrm{lim}} \approx 17.7$; $m^{*} \approx-20.83+5 \log D_{i}+2.5 \log (1+z)$ (Blanton et al. 2001); the dashed lines show the limits, with \pm 0.03 error in $M^{*}$ and $\pm 20 \%$ error in the $k$-correction. The luminosity distance is calculated assuming a world model with $C_{0}=(1.0,0.3,0.7)$. The right plot shows the aperture scale for fiber diameters of 2 ". 1 and 3 ".0. 

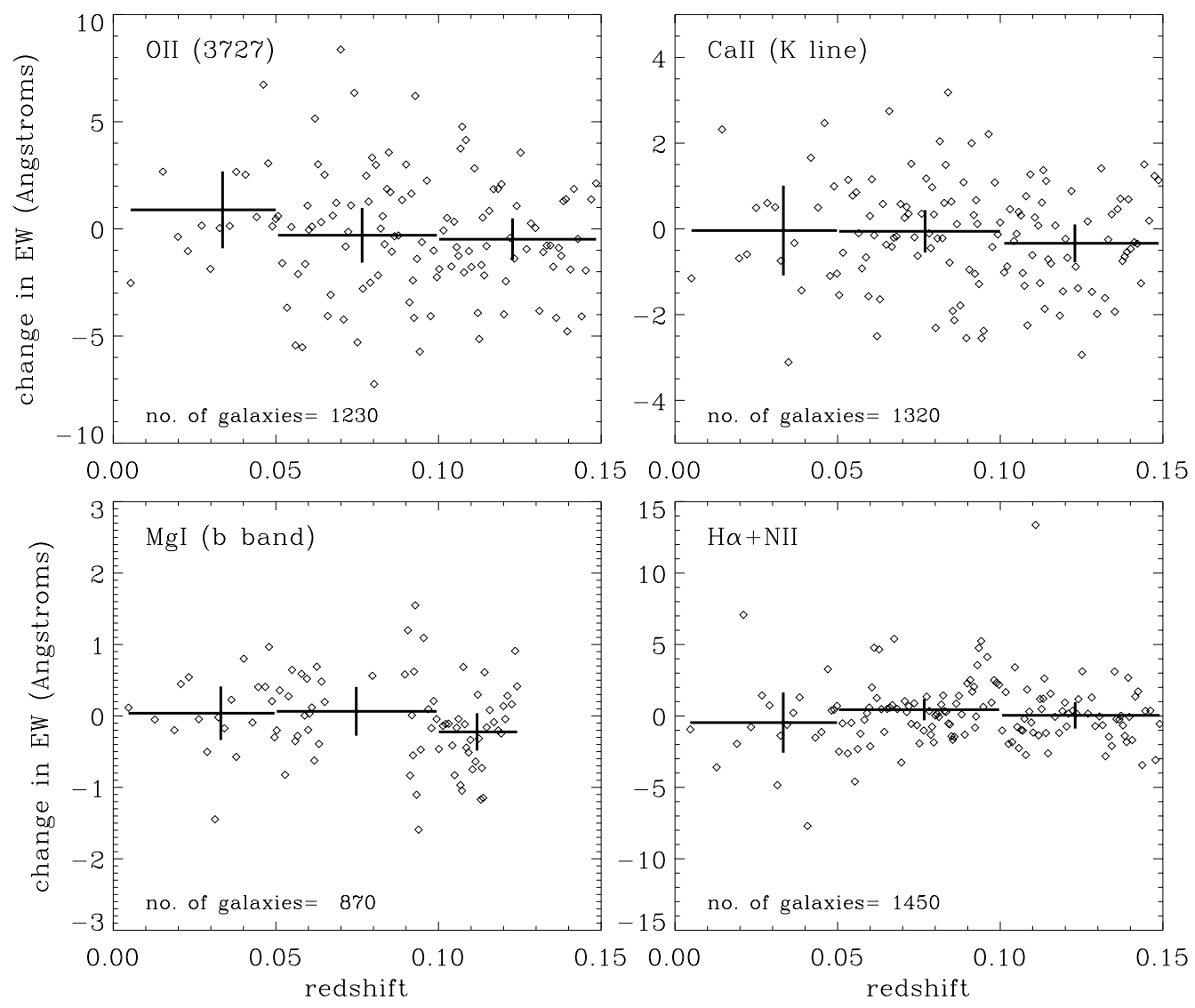

FIG. 11. Change of line EWs- O II, Ca II, Mg I, and $\mathrm{H} \alpha$ - between measurements taken in poor seeing and in good seeing. Each diamond represents the median change of a subsample of 10 galaxies. The lines represent the mean of the subsamples, \pm 3 times the standard error of the mean, divided into three redshift ranges. [See the electronic edition of the Journal for a color version of this figure.]

and evolution? Figure 12 plots the equivalent width of $\mathrm{O}$ II, $\mathrm{H} \delta, \mathrm{CH}, \mathrm{O}$ III, $\mathrm{Mg}$ I, and $\mathrm{Na}$ I as a function of redshift for a complete sample and for a volume-limited sample. These measurements were made on luminosity-weighted, averaged spectra determined from redshift bins of mostly 0.005 in extent. To interpret this figure, we first consider the volumelimited sample (diamonds).

1. In the redshift range $0.05-0.2$, we expect aperture effects to be minimal. Therefore, the change in EW in this range could be primarily due to evolution, and the changes agree qualitatively with a $\beta \sim 3$ scenario. It is not possible to make an accurate comparison, because of the lower resolution of the model spectra. However, qualitatively they agree well: nebular line emission decreases between $z=0.2$ and $z=0.05$; the $\mathrm{CH}$ absorption EW increases; $\mathrm{Mg} \mathrm{I}$ and $\mathrm{Na}$ I absorption increase less; and, not surprisingly, the $\mathrm{H} \delta$ absorption decreases because of a lower fractional contribution of A stars. The solid lines in Figure 12 are fits to these changes: with the $\mathrm{EW} \propto(1+z)^{\beta_{\mathrm{ew}}}$ for the emission lines, and $\mathrm{EW}=\beta_{\mathrm{ew}}^{\prime} z+$ const for the others. These constitute a detection of cosmic evolution. However, we cannot unambiguously separate cosmic SFR evolution from galaxy population evolution, and so we have not used these measurements to constrain the cosmic SFR in this paper.

2. In the redshift range $0.02-0.05$, the change in EW for most of the lines levels off or reverses direction. As described above, we expect the evolutionary changes to continue from $z=0.1$ to $z=0.02$. Our interpretation is that at low redshift the fiber aperture will only see a small part of the galaxy, which for a blue-targeted sample is biased toward more recent star formation; thus, the nebular line emission is higher and the $\mathrm{CH}$ absorption is lower. We note that for an individual extended spiral galaxy, a $2 \mathrm{dF}$ fiber spectrum could be biased toward more quiescent regions (e.g., the bulge) or more star-forming regions. Here, we are strictly referring to the average effect on the measured cosmic spectrum (the median bias may still be toward the bulge). We note also that the nebular line emission may be more sensitive to aperture and selection effects (and we ignore them in the fitting) than features at other wavelengths derived from older stellar populations.

Considering the all-selection sample (crosses) from Figure 12 , it is clear that luminosity selection bias is having a more significant effect than aperture bias, i.e., the difference between not including and including the lower luminosity galaxies in the cosmic spectrum is larger than the difference between using small and large physical apertures on a volume-limited sample, given the above interpretation. This is most evident for the $\mathrm{O}$ II and $\mathrm{O}$ III emission lines, showing that the less luminous galaxies contain a higher fraction of recent star formation. The $2 \mathrm{dFGRS}$ is a magnitude-limited sample, so the luminosity cutoff will rise with redshift. This is illustrated in Figure 10; at $z=0.1$ we are probing $1.5 \mathrm{mag}$ fainter than the Schechter luminosity function break $M^{*}$. For a Schechter function with a faint-end slope of -1.19 (the 2dFGRS value found by Madgwick et al. 2002), this 


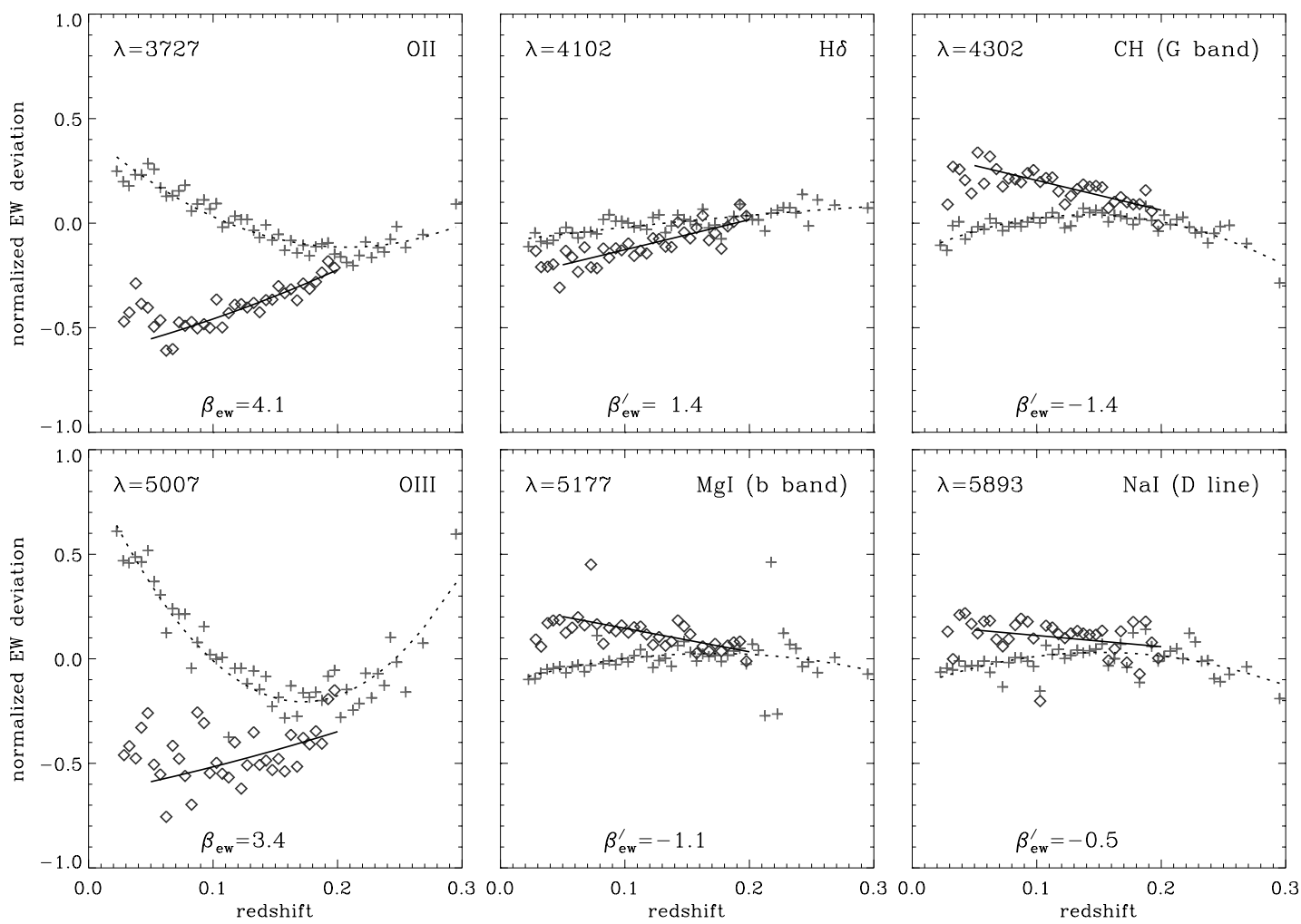

FIG. 12.-Variation of average line EWs- $\mathrm{O}$ II, $\mathrm{H} \delta, \mathrm{CH}, \mathrm{O}$ III, $\mathrm{Mg}$ I, and $\mathrm{Na}$ I-as a function of redshift in the $2 \mathrm{dFGRS}$ data. Crosses: 49 redshift bins between 0.020 and 0.32 , selecting all galaxies. Dotted lines: Quadratic fits. Diamonds: Volume-limited sample of 35 redshift bins between 0.025 and 0.20 , selecting galaxies brighter than a $k$-corrected absolute magnitude of -21 (using $h=0.7$ ). Solid lines: Straight-line fits over the redshift range where cosmic evolution is expected to dominate the measured variations. The normalized deviation is defined as $(E-\langle E\rangle) /\langle E\rangle$, where $E$ is the EW and $\langle E\rangle$ is the mean of the complete sample. Note that the normalization is such that a positive value means an increase in emission for the emission lines (left panels) or an increase in absorption for the others. [See the electronic edition of the Journal for a color version of this figure.]

will encompass about $70 \%$ of the light at $z=0.1$ and $95 \%$ at $z=0.04$, but only $20 \%$ at $z=0.2$.

It should be noted that all magnitude-limited samples are dominated by $M \sim M^{*}$ galaxies. This is a conspiracy between the volume probed and the shape of the Schechter function (see, e.g., Glazebrook 1992 for a discussion of this). Other studies of luminosity density evolution via flux-limited samples will also be affected by this dominance of $M^{*}$ galaxies, so the comparison of our results in $\S 4.3$ is consistent.

Consider our divided redshift ranges, $0.03-0.11$ and $0.11-$ 0.25 . For the lower range, the luminosity selections are less significant (the measured cosmic spectrum samples a significant range of the galaxy luminosity function), but the aperture effects may be biasing the averaged spectra toward more recent star formation. For the higher range, the aperture effects are less significant, but the selection function is clearly missing a higher fraction of recent star formation from the lower luminosity galaxies. However, in general, we have found the results to be robust against choice of redshift range chosen: lower, higher, or combined. In any case, the contours in Figures 4-9 take account of the variation in best-fit parameters between the lower and higher redshift ranges.

\section{DISCUSSION AND CONCLUSIONS}

We have developed a method of determining relative cosmic star formation history based only upon the spectral information. We find consistent results between both lowpass and high-pass spectral information and between physical and empirical parameterizations of the star formation law. If we assume that (1) the averaged 2dFGRS spectra ( $z$ in the range $0.03-0.25$ ) represent the galaxy population as a whole, (2) the stellar populations can be approximated by one chemical evolution scenario, and (3) the IMF, the PEGASE models, and the cosmology are sufficiently accurate, then we can reach the following conclusions.

1. The present-day "cosmic spectrum" $" 16$ is well determined at $z \sim 0.1$ and can only be fitted with models incorporating consistent chemical evolution. Constant metallicity is strongly ruled out. The final metallicity averaged on the luminosity is around $\operatorname{solar}(Z \approx 0.01-0.04)$.

2. The star formation timescale is longer than, or approximately equal to, the infall timescale of gas assembly.

3 . The significant majority of nearby galaxies (weighted by their luminosity) have $z_{\text {form }} \gtrsim 0.65$, where $z_{\text {form }}$ is the redshift at which star formation began.

\footnotetext{
${ }^{16}$ It is interesting to compute the perceived color of the cosmic spectrum to the human eye, using standard color-matching functions CIE (1971, 1986). Integrating these through the cosmic spectrum, we have computed CIE $x y$-values of $0.345,0.345$ which are robust against a choice of redshift bins. This is close to white, which means, that the perceived color can vary significantly depending on the adaption of the eye to varying conditions. With reference to an equal-energy white point (Flat $f_{\lambda} ; x y=0.333,0.333$ ), the color would appear a pale yellow but can appear more orange or bluegreen with respect to other white points (D65, A, respectively).
} 
4. There was a peak in cosmic star formation rate density in the past, which was a factor of at least 3 times the presentday rate. With the physical parameterization, the peak occurs between $z=0.6$ and $z=10$ (using limits of $z_{\text {form }} \leq 32$ and $t_{i} \geq 100 \mathrm{Myr}$ ). With the empirical parameterization, the peak occurs at $z=1$ or $z=5$ (with an instantaneous rise in the SFR at $z=5$ ).

5. Strong upper limits on star formation at high redshift $(\alpha)$ can be obtained if we take inputs on the value of lowredshift star formation $(\beta)$ from other studies (see, e.g., Lilly et al. 1996). If we take $\beta \gtrsim 3$ (i.e., at least 8 times the SFR at $z=1$ relative to that at $z=0$ ), then $\alpha<0.5$ (Salpeter IMF, $\mathscr{C} 1)$. These values are consistent with the no-dust-extinction SFR models of Madau et al. (1998) and rule out the test case of a much higher SFR density at high redshift with a dust opacity that increases rapidly with redshift (their Fig. 7, which has $\beta \approx 3$ and $\alpha \approx 1$ ). The test case is also not consistent with our results for $\mathscr{C} 2$, which has $\alpha<-1$ for $\beta \gtrsim 3$. This upper limit corresponds to a maximum of $65 \%$ and $55 \%$ of the stars forming at $z>1$ in $\mathscr{C} 1$ and $\mathscr{C} 2$, respectively. Correspondingly, if we take $\beta \gtrsim 2$, then a maximum of $80 \%$ and $75 \%$, formed at $z>1$. Note that if there is any significant star formation before $z=5$, this will lower the value of $\alpha$ (or $\beta$ ) required to give a suitable model fit to the presentday cosmic spectrum.

6. Alternatively, if we take the view that star formation was either constant or declining at high redshift (i.e., $\alpha \lesssim 0$ ), then we infer a rise in SFR to redshift unity, $1.5<\beta<5$, consistent with studies of luminosity density evolution.

7. From the 2MASS-2dFGRS measurement of $\Omega_{\text {stars }}$, Cole et al. (2001) concluded that their results were "only consistent with recent determinations of the integrated cosmic star formation if the correction for dust extinction is modest." Here we find again that there cannot be massive amounts of star formation concealed at high redshift by dust extinction. Cole et al.'s analysis compares the $J$ and $K_{S}$ luminosity density derived from $2 \mathrm{dFGRS}$ redshifts and 2MASS photometry with semianalytic models of galaxy/ star formation to derive an integrated stellar mass density today. Our method derives the star formation history empirically, using 2dFGRS spectra and redshifts.

8. From the $b_{J}$ luminosity density of Madgwick et al. (2002) and computing the mass-to-light and SFR-to-light ratios from the PEGASE scenarios, we estimated the present-day stellar mass density and SFR density. Even when the models are restricted to good fits with $\beta \gtrsim 1.5$ and $\alpha \gtrsim-3$, there is still significant degeneracy particularly with metallicity. For cosmology $\mathscr{C} 1(a)$ with the Salpeter (1955) IMF, $\Omega_{\text {stars }} h$ is in the range $0.0020-0.0062$ and $\rho_{\text {SFR }}$ is in the range 0.024-0.065 $h M_{\odot} \mathrm{yr}^{-1} \mathrm{Mpc}^{-3}$; $(b)$ with the Kennicutt (1983) IMF, $\Omega_{\text {stars }} h$ is in the range $0.0013-0.0033$ and $\rho_{\text {SFR }}$ is in the range $0.012-0.055 h M_{\odot} \mathrm{yr}^{-1} \mathrm{Mpc}^{-3}$. For cosmology $\mathscr{C} 2$, the mass-to-light ratios increase and the values for $\Omega_{\text {stars }} h$ are increased by a factor of about 1.2. The SFR-tolight ratios remain approximately the same. These results are consistent with the stellar mass density derived by Cole et al. and with other derivations of the local SFR density (see, e.g., Sullivan et al. 2000).

Finally, we compare our results with two different compilations of cosmic SFR based on the rest-frame UV luminosity density technique and with a compilation of various other techniques. The $\alpha-\beta$ empirical parameterization was fitted to the data sets after conversion to cosmology $\mathscr{C} 1$. The

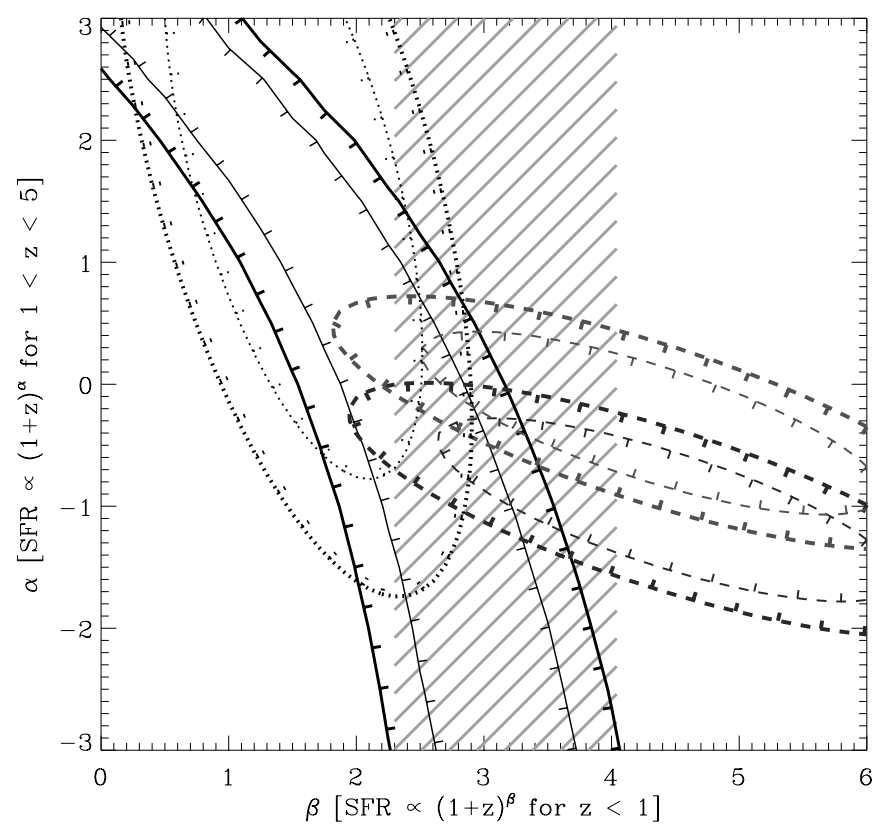

FIG. 13. - Comparison of different cosmic SFR studies: confidence limits in $\alpha$ vs. $\beta$. Solid contours: $2 \sigma$ and $3 \sigma$ confidence limits from this paper with FOM B, $r=1.1$, Salpeter IMF, and $C_{0}=(0.70,0.3,0.7)$. Dashed contours: Limits from a compilation of UV luminosity-density measurements, with a redshift range of 0.2-4.5 (Connolly et al. 1997; Lilly et al. 1996; Madau et al. 1996; Steidel et al. 1999), see Steidel et al.'s Fig. 9. The lower and upper dashed contours are for no-extinction and extinction-corrected data, respectively. Dotted contours: Limits from a compilation of UV measurements, with a $z$ range of 0.0-1.5 (Cowie et al. 1999; Loveday et al. 1992; Sawicki, Lin, \& Yee 1997; Treyer et al. 1998), see Cowie et al.'s Fig. 13. Shaded region: The $1 \sigma$ bootstrap confidence on $\beta$ from the compilation of various techniques by Hogg (2001), except we have excluded the UV measurements. The data include nebular line emission, far-IR, and radio continuum measurements, with a $z$ range of 0.0-1.0 (Flores et al. 1999; Gallego et al. 1995; Glazebrook et al. 1999; Haarsma et al. 2000; Hammer et al. 1997; Hogg et al. 1998; Mobasher et al. 1999; Rowan-Robinson et al. 1997; Tresse \& Maddox 1998)

various contours are shown in Figure 13, with full references quoted in the caption.

For the first UV compilation, we took the 10 data points with error bars from Figure 9 of Steidel et al. (1999) and calculated formal confidence boundaries in $\alpha$ versus $\beta$. The noextinction and extinction-corrected data were considered separately. This compilation promotes a high value of $\beta \sim 4$, with $\alpha$ depending on extinction.

For the second UV compilation, we took nine data points with error bars from Figure 13 of Cowie, Songaila, \& Barger (1999) for the $2800 \AA$ luminosity density, excluding those used in the first compilation. A minimum error of 0.07 in the $\log$ was used to calculate the boundaries. This compilation promotes " a gradual decline" (Cowie et al. 1999) in the UV luminosity density for $z \lesssim 1.5$ with $\alpha=\beta \sim 1.5$.

For the other compilation, we recomputed the bootstrap error from the measurements of $\beta$ given by Hogg (2001), excluding the UV measurements included above. This new value was $\beta=3.2 \pm 0.9$.

A precise interpretation of Figure 13 depends on the accuracy of the errors used in the analysis. Given the analysis presented here, the two UV compilations (taking either extinction model from the first) are consistent at the $3 \sigma$ level with $1.8<\beta<2.9$ and $-1.0<\alpha<0.7$, but not quite consistent at the $2 \sigma$ level. Our results are independently consis- 
tent with these ranges of values but do not constrain the region further. Thus, evolutionary synthesis applied to the 2dFGRS-measured cosmic spectra $(z \sim 0.03-0.25)$ is in concordance with the best-fit results obtained from a variety of rest-frame UV luminosity-density measurements $(z \sim 0-4.5)$.

\subsection{Future Work}

With this technique of primarily using the "high-frequency" spectral information, there are still significant degeneracies in determining the cosmic star formation history. However, combined with luminosity-density methods and with further improvements, there is the potential to obtain more accurate star formation scenarios and to discriminate between IMFs and cosmologies. For example, the separation of galaxies into groups with similar star formation history may help break the degeneracy, i.e., reduce the merging of features between young and old populations. In addition, it may be possible to improve the fit to the spectra by combining models with different chemical evolution scenarios, e.g., weighted combinations of spectra with different $t_{i}, t_{s}$, and $z_{\text {form }}$.

Future large surveys, such as the SDSS main galaxy sample will provide a consistency check with the $2 \mathrm{dFGRS}$ spectra (selected with different effective wavelengths [6200 vs. $4700 \AA$ A], magnitude limits, and aperture diameters; see Fig. 10). With the 2dFGRS spectra (resolution 8-9 A), and especially with the SDSS spectra ( $3-4 \AA)$, it is apparent that higher spectral resolution population synthesis models ( $\sim 2 \AA$ in the optical wavelength range) are needed to maximize the scientific potential of these data sets. Work is currently underway for this purpose.

Both galaxy surveys can also be used to measure the slope of luminosity density variation with redshift in the local universe, which provides another star formation estimate from the same survey. This can accomplished either by using $k$ corrections and determining the luminosity function in the rest frame or by determining the luminosity function in observed wavelength, i.e., the extragalactic background light per unit redshift. These measurements can be used to apply further constraints on the cosmic star formation scenarios and the absolute cosmic comoving star formation rate density.

The 2dF Galaxy Redshift Survey was made possible through the dedicated efforts of the staff of the AngloAustralian Observatory, both in creating the $2 \mathrm{dF}$ instrument and in supporting the survey observations. Karl Glazebrook and Ivan Baldry acknowledge generous funding from the David and Lucille Packard foundation.

\section{REFERENCES}

Abraham, R. G., Ellis, R. S., Fabian, A. C., Tanvir, N. R., \& Glazebrook, K. 1999, MNRAS, 303, 641

Blanton, M. R. et al. 2001, AJ, 121, 2358

CIE, 1971, Publ. 15, Colorimetry (Vienna: Commission Internationale de l'Éclairage)

. 1986, Publ. S002, Standard on Colorimetric Observers (Vienna: Commission Internationale de l'Éclairage)

Cole, S., et al. 2001, MNRAS, 326, 255

Colless, M., Ellis, R. S., Taylor, K., \& Hook, R. N. 1990, MNRAS, 244, 408

Colless, M., et al. 1999, in Looking Deep in the Southern Sky, ed. R. Morganti \& W. J. Couch (Berlin: Springer), 9 2001, MNRAS, 328, 1039

Connolly, A. J., Szalay, A. S., Dickinson, M., SubbaRao, M. U., \& Brunner, R. J. 1997, ApJ, 486, L11

Cowie, L. L., Lilly, S. J., Gardner, J., \& McLean, I. S. 1988, ApJ, 332, L29

Cowie, L. L., Songaila, A., \& Barger, A. J. 1999, AJ, 118, 603

de Bernardis, P., et al. 2000, Nature, 404, 955

de Jong, R. S., \& Lacey, C. 2000, ApJ, 545, 781

Fioc, M., \& Rocca-Volmerange, B. 1997, A\&A, 326, 950

Flores, H., et al. 1999, ApJ, 517, 148

Gallego, J., Zamorano, J., Aragón-Salamanca, A., \& Rego, M. 1995, ApJ, $455, \mathrm{~L} 1$

Glazebrook, K. 1992, Ph.D. thesis, Univ. Edinburgh

Glazebrook, K., Blake, C., Economou, F., Lilly, S., \& Colless, M. 1999, MNRAS, 306, 843

Haarsma, D. B., Partridge, R. B., Windhorst, R. A., \& Richards, E. A. 2000, ApJ, 544, 641

Hammer, F., et al. 1997, ApJ, 481, 49

Hogg, D. W. 2001, ApJ, submitted (astro-ph/0105280)

Hogg, D. W., Cohen, J. G., Blandford, R., \& Pahre, M. A. 1998, ApJ, 504, 622

Hopkins, A. M., Irwin, M. J., \& Connolly, A. J. 2001, ApJ, 558, L31

Hughes, D. H., et al. 1998, Nature, 394, 241

Kennicutt, R. C., Jr. 1983, ApJ, 272, 54 1992, ApJS, 79, 255

Kochanek, C. S., Pahre, M. A., \& Falco, E. E. 2000, ApJ, submitted (astro-ph/0011458)

Kudritzki, R.-P., et al. 2000, ApJ, 536, 19

Lejeune, T., Cuisinier, F., \& Buser, R. 1997, A\&AS, 125, 229

Lewis, I. J., Glazebrook, K. \& Taylor, K. 1998, Proc. SPIE, 3355, 828

Lewis, I. J., et al. 2002, MNRAS, in press (astro-ph/0202175)

Lilly, S. J., Le Fèvre, O., Hammer, F., \& Crampton, D. 1996, ApJ, 460, L1

Loveday, J., Peterson, B. A., Efstathiou, G., \& Maddox, S. J. 1992, ApJ, 390,338

Madau, P., Ferguson, H. C., Dickinson, M. E., Giavalisco, M., Steidel, C. C., \& Fruchter, A. 1996, MNRAS, 283, 1388

Madau, P.. Pozzetti, L. \& Dickinson, M. 1998, ApJ, 498, 106

Maddox, S. J., Efstathiou, G., \& Sutherland, W. J. 1990a, MNRAS, 246 433

. 1996, MNRAS, 283, 1227

Maddox, S. J., Sutherland, W. J., Efstathiou, G., \& Loveday, J. 1990b, MNRAS, 243, 692

Maddox, S., et al. 1998, in Large-Scale Structure: Tracks and Traces, ed. V. Müller, S. Gottlöber, J. P. Mücket, \& J. Wambsganss (Singapore: World Scientific), 91

Madgwick, D. S., et al. 2002, MNRAS, in press (astro-ph/0107197)

Mobasher, B., Cram, L., Georgakakis, A., \& Hopkins, A. 1999, MNRAS, 308, 45

Pettini, M., Kellogg, M., Steidel, C. C., Dickinson, M., Adelberger, K. L., \& Giavalisco, M. 1998, ApJ, 508, 539

Rowan-Robinson, M., et al. 1997, MNRAS, 289, 490

Salpeter, E. E. 1955, ApJ, 121, 161

Sawicki, M. J., Lin, H., \& Yee, H. K. C. 1997, AJ, 113, 1

Schechter, P. L. 1976, ApJ, 203, 297

Silk, J. 1999, PASP, 111, 258

Steidel, C. C., Adelberger, K. L., Giavalisco, M., Dickinson, M., \& Pettini, M. 1999, ApJ, 519,

Sullivan, M., Treyer, M. A., Ellis, R. S., Bridges, T. J., Milliard, B., \& Donas, J. 2000, MNRAS, 312, 442

Taylor, K., \& Gray, P. 1990, Proc. SPIE, 1236, 290

Tresse, L., \& Maddox, S. J. 1998, ApJ, 495, 691

Treyer, M. A., Ellis, R. S., Milliard, B., Donas, J., \& Bridges, T. J. 1998, MNRAS, 300, 303

Woosley, S. E., \& Weaver, T. A. 1995, ApJS, 101, 181 